\title{
Long-term use of selective digestive decontamination in an ICU highly endemic for bacterial resistance
}

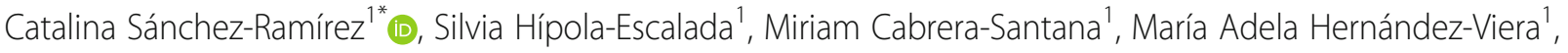 \\ Liliana Caipe-Balcázar ${ }^{1}$, Pedro Saavedra ${ }^{2}$, Fernando Artiles-Campelo ${ }^{3}$, Nayra Sangil-Monroy ${ }^{4}$, \\ Carlos Federico Lübbe-Vázquez ${ }^{1}$ and Sergio Ruiz-Santana ${ }^{1}$
}

\begin{abstract}
Background: We examined whether long-term use of selective digestive tract decontamination (SDD) was effective in reducing intensive care unit (ICU)-acquired infection and antibiotic consumption while decreasing colistin-, tobramycin-, and most of the antibiotic-resistant colonization rates in a mixed ICU with a high endemic level of multidrug-resistant bacteria (MDRB).

Methods: In this cohort study, which was conducted in a 30-bed medical-surgical ICU, clinical outcomes before (1 year, non-SDD group) and after (4 years) implementation of SDD were compared. ICU patients who were expected to require tracheal intubation for $>48$ hours were given a standard prophylactic SDD regimen. Oropharyngeal and rectal swabs were obtained on admission and once weekly thereafter.

Results: ICU-acquired infections occurred in 110 patients in the non-SDD group and in 258 in the SDD group. A significant $(P<0.001)$ reduction of infections caused by MDRB (risk ratio [RR], $0.31 ; 95 \% \mathrm{Cl}, 0.23-0.41$ ) was found after SDD and was associated with low rates of colistin- and tobramycin-resistant colonization. Colistin- and tobramycinacquired increasing rate of ICU colonization resistance by 1000 days, adjusted by the rate of resistances at admission, was nonsignificant $(0.82 ; 95 \% \mathrm{Cl}, 0.56$ to $1.95 ; 1.13 ; 95 \% \mathrm{Cl}, 0.75$ to 1.70 , respectively). SDD was also a protective factor for ICU-acquired infections caused by MDR gram-negative pathogens and Acinetobacter baumannii in the multivariate analysis. In addition, a significant $(P<0.001)$ reduction of ventilator-associated pneumonia (VAP) (RR, $0.43 ; 95 \% \mathrm{Cl}, 0.32-0.59$ ) and secondary bloodstream infection (BSI) $(\mathrm{RR}, 0.35 ; 95 \% \mathrm{Cl}, 0.24-0.52)$ was found. A decrease in antibiotic consumption was also observed.

Conclusions: Treatment with SDD during 4 years was effective in an ICU setting with a high level of resistance, with clinically relevant reductions of infections caused by MDRB, and with low rates of colistin- and tobramycin-resistant colonization with nonsignificant increasing rate of ICU colonization resistance by 1000 days, adjusted by the rate of resistances at ICU admission. In addition, VAP and secondary BSI rates were significantly lower after SDD. Notably, a decrease in antimicrobial consumption was also observed.
\end{abstract}

Keywords: Selective digestive decontamination, Drug resistance, ICU-acquired infection, Ventilator-associated pneumonia, Multidrug-resistant pathogens, Bloodstream infection, Colistin, Tobramycin

\footnotetext{
* Correspondence: catalinasanchezramirez@gmail.com

${ }^{1}$ Intensive Care Unit, Hospital Universitario de Gran Canaria Dr. Negrín, Las

Palmas de Gran Canaria, La Ballena s/n, E-35010 Las Palmas, Spain

Full list of author information is available at the end of the article
}

(c) The Author(s). 2018 Open Access This article is distributed under the terms of the Creative Commons Attribution 4.0 International License (http://creativecommons.org/licenses/by/4.0/), which permits unrestricted use, distribution, and reproduction in any medium, provided you give appropriate credit to the original author(s) and the source, provide a link to the Creative Commons license, and indicate if changes were made. The Creative Commons Public Domain Dedication waiver (http://creativecommons.org/publicdomain/zero/1.0/) applies to the data made available in this article, unless otherwise stated. 


\section{Background}

Selective digestive decontamination (SDD) is a prophylactic treatment for critically ill patients that is based on an oropharyngeal paste and enteral suspension containing antimicrobials, usually tobramycin, colistin, and an antifungal as well as an intravenous antibiotic, administered during the first 4 days of intensive care unit (ICU) admission (usually a second-generation cephalosporin). The aim of SDD is to prevent or eradicate, if present, the oropharyngeal and intestinal abnormal carriage of potentially pathogenic microorganisms, such as aerobic gram-negative bacilli (AGNB), methicillin-sensitive Staphylococcus aureus, and yeasts, in patients at risk for nosocomial infections $[1,2]$. Once a patient has been successfully decolonized, the unaffected anaerobic flora would offer prevention against new colonization with potential pathogenic microorganisms. In critically ill patients, SDD has been proven to prevent severe infections [1-3] and to reduce mortality [3, 4], particularly in settings with a low prevalence of multidrug-resistant bacteria. However, the use of SDD is still a matter of debate, largely because of concerns that it may promote the emergence of antibiotic-resistant strains $[5,6]$. Also, the effect of SDD in ICUs with endemic circulation of multidrug-resistant gram-negative bacilli MDR-GNB) remains controversial [7, 8]. We investigated whether long-term use of SDD was efficacious in reducing ICU-acquired MDR-GNB infection and also sought to determine its effect, including colistin- and tobramycin-resistant colonization as well as other nosocomial infections and subsequent antibiotic consumption, in a mixed ICU with a high endemic level of multidrug-resistant bacteria (MDRB).

\section{Methods}

\section{Study design and patients}

We conducted a prospective cohort study in a 30-bed medical-surgical ICU of an acute care tertiary hospital in Las Palmas de Gran Canaria, Canary Islands, Spain. All consecutive patients admitted to the ICU between September 1, 2010, and September 30, 2015, were included. They were grouped into two consecutive cohorts before and after implementation of SDD. Data of both cohorts were collected prospectively. Patients admitted between September 1, 2010, and September 30, 2011, were included in the non-SDD cohort, and patients admitted between October 1, 2011, and September 30, 2015, were included in the SDD cohort. Since October 1, 2011, SDD measures have been systematically applied to all ICU patients expected to require tracheal intubation for more than 48 hours (SDD cohort). SDD was started when the "Pneumonia Zero" project began to be implemented among Spanish ICUs. In the "Pneumonia Zero" project, SDD was a highly recommended component of the ventilator-associated pneumonia (VAP) prevention bundle [9]. The primary objective was to compare outcome measures between the non-SDD and SDD cohorts.

\section{SDD protocol}

SDD was started on the day of tracheal intubation and was given throughout the length of the ICU stay and until discharge from the ICU. Patients were treated three times daily with $1 \mathrm{~g}$ of an oral paste applied to the oral cavity. The composition per $1 \mathrm{~g}$ was $20 \mathrm{mg}$ of $2 \%$ colistin, $30 \mathrm{mg}$ of $3 \%$ tobramycin, and $20 \mathrm{mg}$ of $2 \%$ nystatin. The patients also received a 14-ml suspension containing $140 \mathrm{mg}$ of $1 \%$ colistin, $180 \mathrm{mg}$ of $2 \%$ tobramycin, and $453.6 \mathrm{mg}$ of $3.2 \%$ nystatin [10], which was administered into the gut through a nasogastric tube. In tracheostomized patients, the oral paste was also applied on the skin surrounding the tracheostomy three times daily. Enteral vancomycin, $40 \mathrm{mg}$ of $4 \%$ oropharyngeal paste, and $700 \mathrm{mg}$ of vancomycin in digestive solution were added at the same 8-hour interval to all methicillin-resistant Staphylococcus aureus (MRSA) carriers, as well as to patients referred from elsewhere until MRSA noncarrier status was documented [11]. All patients received systemic cefotaxime, 1 g every 8 hours, during the first 4 days of SDD therapy, except patients with infections on admission, who were treated with their antibiotics.

\section{Endpoints}

The primary endpoints of the study were the incidence of ICU-acquired infection caused by MDRB, the evolution of colistin- and tobramycin-resistant colonization, and the clinical impact of SDD on MDRB infections. Secondary endpoints were VAP, central line-associated primary bloodstream infection (CLABSI), secondary bloodstream infection (BSI), urinary tract infection, and antibiotic consumption.

\section{Study procedures and definitions}

Surveillance samples from the throat, rectum, tracheostomy, and pressure sores were collected on ICU admission and once weekly thereafter. Diagnostic samples from tracheal aspirates, peripheral blood, urine, or wounds were obtained at the physician's discretion. Antimicrobial susceptibility testing was performed with the VITEK-2 system (bioMérieux, Inc., Durham, NC, USA) [12], with breakpoints defined according to the Clinical and Laboratory Standards Institute [13] and the European Committee on Antimicrobial Susceptibility Testing [14] guidelines. Infections caused by MDRB included the following:

1. Enterobacteriaceae spp. resistant to ceftazidime and/or aminoglycosides and/or ciprofloxacin with 
extended-spectrum $\beta$-lactamase (ESBL) producing bacteria

2. Pseudomonas aeruginosa resistant to ceftazidime and/or aminoglycosides and/or ciprofloxacin and/or imipenem

3. MRSA

4. Any strain of Acinetobacter spp. resistant to carbapenems

5. Gram-negative bacteria resistant to three or more antimicrobial families

6. Clostridium difficile

7. Vancomycin-resistant Enterococcus spp.

Imported MDRB infection was considered when cultures of surveillance or diagnostic samples were positive within 48 hours of ICU admission. ICU-acquired MDRB infection was defined as isolation of a new strain that was not recovered in any of the samples taken during the first 48 hours of admission. Also, secondary endogenous infections were those preceded by gastrointestinal carriage of MDRB with identical antibiotic susceptibility patterns and exogenous infections when the infecting MDRB was isolated in diagnostic samples without previous colonization [15].

ICU-acquired infections were collected from the ENVIN-HELICS registry (National Nosocomial Infection Surveillance Study-Hospitals in Europe Link for Infection Control through Surveillance), which is a nationwide ongoing multicenter data collection system designed to record invasive device-related infections in ICU patients (http:// hws.vhebron.net/envin-helics/). Diagnostic criteria established by the ENVIN-HELICS project were used [16]. The diagnosis of VAP included the following:

1. Sequential chest $x$-rays or computed tomographic (CT) scans with an image suggestive of pneumonia (two or more radiographs or CT scans in the presence of underlying cardiac or pulmonary disease)

2. Fever $\left(>38{ }^{\circ} \mathrm{C}\right)$ and/or leukocytosis $(\geq 12,000$ white blood cells $\left.[\mathrm{WBC}] / \mathrm{mm}^{3}\right)$ or leukopenia $(\leq 4000$ $\mathrm{WBC} / \mathrm{mm}^{3}$ )

3. At least one of the following:

a. New-onset purulent sputum or change in the characteristics of sputum

b. Cough, dyspnea, or tachycardia

c. Rales or bronchial breath sounds on auscultation, ronchi, wheezing

d. Worsening gas exchange

Other infections were diagnosed according to the Centers for Disease Control and Prevention definitions [17] when applicable to ICU patients.

\section{Ethics}

Our ICU participated in the ENVIN-HELICS national registry, and we used this registry for prospective data collection during the study [18]. Baseline data collection started in 2010. The ENVIN-HELICS registry was approved by the ethics committees of the majority of participating ICUs and was declared a registry of healthcare interest by the Spanish Ministry of Health, Social Services and Equality in 2014. The ENVIN-HELICS registry was also approved by our hospital's ethics committee. We applied SDD in the context of the Spanish national "Pneumonia Zero" project [9], the framework for implementing SDD, which is supported by the Spanish Ministry of Health, Social Policy and Equality through a contract with the Spanish Society of Critical Care Medicine and Coronary Units (number 0100/ 2010/0784). The study protocol was approved by the Clinical Research Ethics Committee of Hospital del Mar (Barcelona, Spain), which was the national reference committee.

\section{Statistical analysis}

Categorical variables are expressed as frequencies and percentages, and quantitative variables are expressed as mean \pm SD or median and IQR (25th-75th percentiles) as appropriate. Percentages were compared with the $X^{2}$ test, means with Student's $t$ test, and medians with the Wilcoxon test for independent data. Statistically significant variables in the univariate analysis were introduced in a multivariate logistic regression model, with selection of variables based on a complete enumeration algorithm and the Bayes information criterion. The models were summarized as coefficients $(\beta)$, SE, $P$ values (likelihood ratio test), and ORs, which were estimated by $95 \%$ CIs.

For each ICU-acquired infection, the incidence per 1000 days of exposure in each cohort and the corresponding relative risks (RRs) were obtained by Poisson regression analysis. Specifically, for the $i$ th cohort determined by hospital, year, and month, we denote by $m_{i}$ the number of events and by $d_{i}$ the number of days of exposition (for all patients). A random effects Poisson model [19] was considered, which assumes that, $m_{i} \sim \operatorname{Poisson}\left(v_{i} \mu_{i}\right)$ is:

$$
\log \mu_{i}=\log d_{i}+\alpha+\beta \cdot S D D_{i}
$$

where $v_{1}, \ldots, v_{k}$ are continuous positive valued $i \mathrm{dd}$ random variables such that $E\left[v_{i}\right]=1$ and $\operatorname{var}\left(v_{i}\right)=\tau$. $S D D$ is $1 / 0$ according presence/absence of SDD. The parameter $\tau$ is the overdispersion. The RR deduced from the model is $R R=\exp \beta$. The model was estimated by the likelihood method and summarized by the RRs, which were estimated by $95 \%$ CIs. Statistical significance was set at $P \leq 0.05$. Data were analyzed using the $\mathrm{R}$ package, version 3.3.1 (R Development Core Team, 2016) [20].

\section{Results}

During the 5-year study period, 3948 critically ill patients were admitted to the ICU, and ICU-acquired 
infection (VAP, CLABSI, secondary BSI, urinary tract infection) was diagnosed in 368 of them (7.8\%). Of a total of 994 patients admitted to the ICU between September 2010 and September 2011, 110 patients had ICU-acquired infection in the non-SDD cohort. Of the 3948 patients admitted between October 2011 and September 2015, SDD was administered to 1998 (50.6\%), and 258 developed an ICU-acquired infection (SDD cohort) (Fig. 1). No complications related to the use of SDD were recorded.

Results of univariate analysis are shown in Table 1. Demographic data and the distribution of most variables were similar in both cohorts. In the non-SDD cohort, the percentage of patients with chronic obstructive pulmonary disease and CLABSI was significantly lower than in the SDD cohort. However, we observed significantly lower rates of infections caused by MRDB, including Acinetobacter spp., other GNB and ESBL-producing multidrug-resistant bacteria, VAP, and secondary BSI, in the SDD cohort than in the non-SDD cohort. A significantly higher number of patients with CLABSI in the SDD cohort than in the non-SDD cohort was found. ICU-acquired infections caused by $C$. difficile or vancomycin-resistant Enterococcus spp. did not occur. In the multivariate analysis, SDD was found to be a protective factor against ICU-acquired infections caused by Acinetobacter spp. and MDR-GNB (Table 2). In the multivariate logistic regression model for MDRB

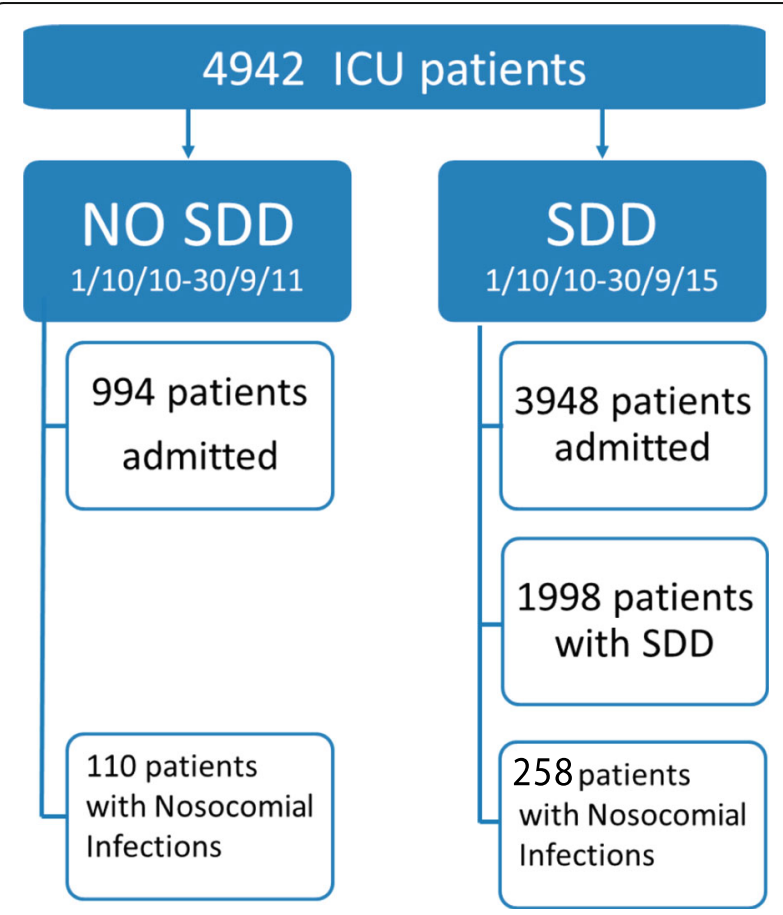

Fig. 1 Patient flowchart. SDD Selective digestive tract decontamination infection, renal replacement therapy (OR, 2.130; 95\% CI, 1.346-3.372; $P=0.001$ ) was an independent risk factor for MDRB infection, whereas SDD was a protective factor (OR, 0.491; 95\% CI, 0.305-0.790; $P<0.001$ ).

Treatment with SDD was associated with a significant reduction of the RR for ICU-acquired infections caused by MDRB, VAP, and secondary BSI (Table 3 ). The probabilities of acquiring infections caused by MDRB, VAP, and secondary BSI were $69 \%, 57 \%$, and $66 \%$ lower, respectively, in the SDD cohort than in the non-SDD cohort.

The consumption of nine antimicrobial agents commonly used in critically ill patients for treating MDRB, expressed as defined daily dose per 100 bed-days in the ICU, also showed a marked reduction after implementation of the SDD prophylactic strategy (Table 4). During the study period, other maneuvers directed toward reducing the use of antimicrobials were not applied.

Of a total of 3948 patients admitted to the ICU during the 4-year period of implementation of the SDD treatment, 285 showed surveillance samples colonized by colistin- or tobramycin-resistant pathogens. As shown in Table 5, there were increases of colonization resistance to colistin and tobramycin at ICU admission. Also, as shown in Table 5, the estimated rates adjusted to 100 patients with SDD decreased in the fourth year for tobramycin-resistant colonization and increased from 1.6 to 1.8 for colistin-resistant colonization in the third and fourth years of the study. The colistin- and tobramycin-acquired increasing rates of colonization resistance in the ICU by 1000 days and adjusted by the rate of resistances at admission were 0.82 (95\% CI, 0.56 to 1.95 ; not statistically significant [NS]) and 1.13 (95\% CI, 0.75 to 1.70 ; NS), respectively. The highest estimated rates of colistin- and tobramycin-resistant colonization by 1000 days in the ICU were 1.2 and 1.1 per 1000 days of ICU stay, respectively (Table 6). A summary of the study findings is shown in Fig. 2.

\section{Discussion}

The main finding of the present study is a significant reduction in the incidence of infections caused by MDRB, including Acinetobacter spp., and other GNBand ESBL-producing pathogens after 4 years of implementation of SDD in the daily care of ICU patients. Additionally, low rates of colistin- and tobramycin-resistant colonization were also observed in surveillance samples, with no significant increasing rate of ICU colonization resistance, by 1000 days, adjusted by the rate of resistances at ICU admission. In addition, VAP and secondary BSI infection rates declined. These findings were associated with a reduction in antibiotic consumption, which is a remarkable aspect of the present results. 
Table 1 Results of univariate analysis

\begin{tabular}{|c|c|c|c|}
\hline Variables & $\begin{array}{l}\text { Non-SDD cohort } \\
(n=110)\end{array}$ & $\begin{array}{l}\text { SDD cohort } \\
(n=258)\end{array}$ & $P$ value \\
\hline Male sex & $74(67.3)$ & $166(64.3)$ & 0.589 \\
\hline Age, years, mean \pm SD & $59.5 \pm 15.8$ & $60.7 \pm 16.4$ & 0.539 \\
\hline APACHE II score on admission, mean \pm SD & $21.2 \pm 7.7$ & $22.0 \pm 7.7$ & 0.345 \\
\hline Glasgow Coma Scale score, median (IQR) & $15(8-15)$ & $14.5(8-15)$ & 0.098 \\
\hline Diagnosis on ICU admission & & & 0.289 \\
\hline Medical & 79 (71.8) & $190(73.6)$ & \\
\hline Scheduled surgery & $10(9.1)$ & $33(12.8)$ & \\
\hline Emergency surgery & $21(19.1)$ & $35(13.6)$ & \\
\hline Septic response & & & 0.399 \\
\hline Sepsis & $57(52.8)$ & $110(45.45)$ & \\
\hline Septic shock & $51(47.2)$ & $132(54.55)$ & \\
\hline Prior surgery & $18(16.4)$ & $37(14.3)$ & 0.618 \\
\hline Urgent surgery & $34(30.9)$ & $70(27.1)$ & 0.461 \\
\hline Trauma patients & $17(15.5)$ & $31(12.0)$ & 0.370 \\
\hline Current smokers & $21(19.1)$ & $31(27.4)$ & 0.141 \\
\hline \multicolumn{4}{|l|}{ Underlying illness } \\
\hline Diabetes mellitus & $34(30.9)$ & $86(33.3)$ & 0.650 \\
\hline Coronary artery disease & $19(17.3)$ & $45(17.4)$ & 0.969 \\
\hline Chronic liver disease & $6(5.5)$ & $18(7.0)$ & 0.588 \\
\hline Chronic obstructive lung disease & $9(8.2)$ & $43(16.7)$ & 0.032 \\
\hline Solid neoplasm & $10(9.1)$ & $26(10.1)$ & 0.771 \\
\hline Chronic renal failure & $40(36.4)$ & $56(21.7)$ & 0.003 \\
\hline Renal replacement therapy & $34(30.9)$ & $91(35.3)$ & 0.419 \\
\hline Parenteral nutrition & $26(23.6)$ & $50(19.4)$ & 0.356 \\
\hline Immunosuppression & $8(7.3)$ & $22(8.5)$ & 0.687 \\
\hline Malnutrition & $12(10.9)$ & $24(9.3)$ & 0.635 \\
\hline \multicolumn{4}{|l|}{ ICU-acquired infection } \\
\hline VAP & $59(53.6)$ & $102(39.5)$ & 0.013 \\
\hline CLABSI & $26(23.6)$ & $106(41.1)$ & 0.001 \\
\hline Secondary BSI & $31(28.2)$ & $47(18.2)$ & 0.023 \\
\hline Urinary tract infection & $29(26.4)$ & $73(28.3)$ & 0.705 \\
\hline \multicolumn{4}{|l|}{ Infections caused by MDRB } \\
\hline Gram-negative bacilli & $12(10.9)$ & $8(3.1)$ & 0.002 \\
\hline Acinetobacter spp. & $13(11.8)$ & $3(1.2)$ & $<0.001$ \\
\hline ESBL-producing MDRB & $38(34.5)$ & $62(24.0)$ & 0.038 \\
\hline Pseudomonas aeruginosa & $10(9.1)$ & $23(8.9)$ & 0.957 \\
\hline Methicillin-resistant Staphylococcus aureus & $4(3.6)$ & $5(1.9)$ & 0.460 \\
\hline ICU stay, days, median (IQR) & $28(16-45)$ & $33(17-50)$ & 0.192 \\
\hline ICU mortality & $36(32.7)$ & $85(33.2)$ & 0.929 \\
\hline
\end{tabular}

Abbreviations: SDD Selective digestive tract decontamination, ICU Intensive care unit, APACHE Acute Physiology and Chronic Health Evaluation, VAP Ventilator-associated pneumonia, CLABSI Central line-associated bloodstream infection, BSI Bloodstream infection, MDRB Multidrug-resistant bacteria, ESBL Extended-spectrum $\beta$-lactamase

Data are expressed as frequency and percent unless otherwise stated 
Table 2 Results of multivariate logistic regression analysis for selective digestive tract decontamination

\begin{tabular}{lll}
\hline Variable & $P$ value & OR (95\% Cl) \\
\hline CLABSI & 0.003 & $2.218(1.307$ to 3.764$)$ \\
Acinetobacter spp. & $<0.001$ & $0.091(0.025$ to 0.329$)$ \\
MDR-GNB & 0.001 & $0.204(0.079$ to 0.527$)$
\end{tabular}

CLABSI Central line-associated bloodstream infection, MDR-GNB Multidrugresistant gram-negative bacilli

We found a significant reduction of ICU-acquired infections caused by MDR-GNB following SDD in our ICU with a high level of antibiotic resistance before implementation of the SDD strategy. There is limited information on the effects of SDD in settings with high levels of MDRB. Four observational studies [7, 21-23] and one small randomized controlled trial [8] have been performed in ICUs where MDR-GNB were endemic or that had an outbreak of certain species of MDR-GNB. In these studies, SDD was applied either as a systematic treatment [21-23] or as a targeted approach for identified carriers [7, 8]. Most of these previous studies examined the effect of SDD on elimination or persistence of carriage of resistant strains, but ecological outcomes were not reported. Moreover, heterogeneity regarding settings and designs prevented clear interpretation of the findings; in fact, SDD was
Table 4 Antibiotic consumption during the study period

\begin{tabular}{|c|c|c|c|c|c|}
\hline \multirow[t]{2}{*}{ Drug } & \multirow{2}{*}{$\begin{array}{l}\text { Non- } \\
\text { SDD } \\
\text { period } \\
\text { (1 year) }\end{array}$} & \multicolumn{4}{|c|}{ SDD period (4 years) } \\
\hline & & 1st year & 2nd year & 3 rd year & 4th year \\
\hline Levofloxacin & 59.01 & 38.10 & 50.79 & 43.96 & 13.89 \\
\hline Meropenem & 43.09 & 32.46 & 32.30 & 27.9 & 11.10 \\
\hline Imipenem & 25.08 & 10.20 & 12.57 & 6.06 & 3.15 \\
\hline Colistin & 19.17 & 10.78 & 12.13 & 4.98 & 0.43 \\
\hline Vancomycin & 7.23 & 4.95 & 6.96 & 6.56 & 2.47 \\
\hline Tobramycin & 10.32 & 3.69 & 1.89 & 1.87 & 0.55 \\
\hline Amikacin & 3.13 & 4.28 & 3.10 & 3.08 & 2.47 \\
\hline Ceftazidime & 7.29 & 5.48 & 5.12 & 10.93 & 5.80 \\
\hline Ciprofloxacin & 9.61 & 12.85 & 8.50 & 8.62 & 8.45 \\
\hline Cefotaxime & 6.01 & 22.6 & 22.3 & 22.7 & 22.7 \\
\hline
\end{tabular}

SDD Selective digestive tract decontamination

Data are expressed as defined daily dose per 100 bed-days in the intensive care unit

found to be useful in three studies [7, 21, 23] and failed in two of them [8, 22]. Brun-Buisson et al. [7] reported that SDD reduced colonization or carrier status and infection during an outbreak of ESBL-producing Klebsiella pneumoniae. Our study confirms that SDD can be useful in an environment with high levels of MDR-GNB.

Table 3 Intensive care unit-acquired infection rates

\begin{tabular}{|c|c|c|c|c|}
\hline & Non-SDD cohort $(n=110)$ & SDD cohort $(n=258)$ & $P$ value & Risk ratio $(95 \% \mathrm{Cl})$ \\
\hline \multicolumn{5}{|l|}{ VAP/MV days } \\
\hline Number of VAP & 63 & 110 & $<0.001$ & $0.437(0.320$ to 0.595$)$ \\
\hline Days of MV & 6112 & 24,432 & & \\
\hline VAP/1000 MV days & 10.3 & 4.5 & & \\
\hline \multicolumn{5}{|l|}{ Urinary tract infection/urinary catheter days } \\
\hline Number of urinary tract infections & 33 & 97 & 0.110 & $0.725(0.488$ to 1.076$)$ \\
\hline Days of indwelling urinary catheter & 8707 & 35,312 & & \\
\hline Urinary infections/1000 catheter days & 3.79 & 2.75 & & \\
\hline \multicolumn{5}{|l|}{ CLABSI/CVC days } \\
\hline Number of CLABSI & & & 0.802 & $1.056(0.690$ to 1.615$)$ \\
\hline Days of CVC & 7249 & 30,631 & & \\
\hline CLABSI/1000 CVC days & 3.59 & 3.9 & & \\
\hline \multicolumn{5}{|l|}{ Secondary BSI/ICU days } \\
\hline Number of secondary BSI & 43 & 57 & $<0.001$ & 0.349 (0.237 to 0.516$)$ \\
\hline ICU days of stay & 9176 & 37,857 & & \\
\hline Secondary BSI/1000 ICU days & 4.69 & 1.64 & & \\
\hline \multicolumn{5}{|l|}{ MDRB/ICU days } \\
\hline Number of MDRB infections & 88 & 112 & $<0.001$ & 0.308 (0.233 to 0.408$)$ \\
\hline ICU days of stay & 9176 & 37,857 & & \\
\hline MDRB infections/1000 ICU days & 9.59 & 2.96 & & \\
\hline
\end{tabular}

Abbreviations: SDD Selective digestive tract decontamination, VAP Ventilator-associated pneumonia, MV Mechanical ventilation, CLABSI Central line-associated bloodstream infection, CVC Central venous catheter, BSI Bloodstream infection, MDRB Multidrug-resistant bacteria 
Table 5 Colonization in surveillance samples by colistin- and tobramycin-resistant pathogens

\begin{tabular}{|c|c|c|c|c|c|}
\hline \multirow[t]{3}{*}{ Variables } & \multicolumn{5}{|c|}{ SDD period (between October 2011 and September 2015) } \\
\hline & Total & 1st year & 2nd year & 3rd year & 4th year \\
\hline & $(n=285)$ & $(n=59)$ & $(n=56)$ & $(n=69)$ & $(n=101)$ \\
\hline Male sex, \% & 66.7 & 67.8 & 71.4 & 60.9 & 67.2 \\
\hline Age, years, mean \pm SD & $60.7 \pm 15.0$ & $56.2 \pm 14.4$ & $61.0 \pm 16.0$ & $61.3 \pm 12.4$ & $62.4 \pm 16.1$ \\
\hline Total patients & 3948 & 1067 & 1069 & 851 & 961 \\
\hline Patients with SDD & 1998 & 522 & 381 & 430 & 665 \\
\hline \multicolumn{6}{|l|}{ Colistin } \\
\hline Resistance at ICU admission & $113(39.6)$ & $5(8.5)$ & $17(30.4)$ & $30(43.5)$ & $61(60.4)$ \\
\hline Development of resistance & $30(10.5)$ & $3(5.1)$ & $8(14.3)$ & $7(10.1)$ & $12(11.9)$ \\
\hline \multicolumn{6}{|l|}{ Observed (at ICU admission) } \\
\hline Rate/100 patients & 2.86 & 0.47 & 1.59 & 3.53 & 6.35 \\
\hline Rate/100 patients SDD & 5.66 & 0.96 & 4.46 & 6.98 & 9.17 \\
\hline \multicolumn{6}{|l|}{ Estimated (acquired in ICU) } \\
\hline Rate/100 patients & 0.76 & 0.28 & 0.75 & 0.82 & 1.25 \\
\hline Rate/100 patients SDD & 1.5 & 0.57 & 2.1 & 1.63 & 1.8 \\
\hline \multicolumn{6}{|l|}{ Tobramycin } \\
\hline Resistance at ICU admission & $151(52.9)$ & $17(6.0)$ & $32(11.2)$ & $34(11.9)$ & $68(23.9)$ \\
\hline Development of resistance & $30(10.5)$ & $1(0.4)$ & $3(1.1)$ & $15(5.3)$ & $11(3.9)$ \\
\hline \multicolumn{6}{|l|}{ Observed (at ICU admission) } \\
\hline Rate/100 patients & 3.82 & 1.59 & 2.99 & 3.99 & 7.08 \\
\hline Rate/100 patients SDD & 7.56 & 3.26 & 8.4 & 7.91 & 10.23 \\
\hline Estimated (acquired in ICU) & - & & & & \\
\hline Rate/100 patients & 0.76 & 0.09 & 0.28 & 1.76 & 1.14 \\
\hline Rate/100 patients SDD & 1.5 & 0.19 & 0.79 & 3.49 & 1.65 \\
\hline
\end{tabular}

ICU Intensive care unit, SDD Selective digestive tract decontamination

Table 6 Evolution of rates of resistance to colistin and tobramycin in ICU, by 1000 days

\begin{tabular}{|c|c|c|c|c|c|}
\hline & \multirow[t]{3}{*}{ Resistance } & \multicolumn{4}{|l|}{ Period } \\
\hline & & 1st year & 2nd year & 3rd year & 4th year \\
\hline & & $(n=59)$ & $(n=56)$ & $(n=69)$ & $(n=101)$ \\
\hline Patient-days & & 9228 & 8583 & 10,731 & 9315 \\
\hline \multirow[t]{4}{*}{ Colistin } & At admission & $5(8.5)$ & $17(30.4)$ & $30(43.5)$ & $61(60.4)$ \\
\hline & Acquired in ICU & $3(5.1)$ & $8(14.3)$ & $7(10.1)$ & $12(11.9)$ \\
\hline & Acquired in ICU, by 1000 days & 0.325 & 0.932 & 0.652 & 1.288 \\
\hline & $\begin{array}{l}\text { Acquired in ICU, by } 1000 \text { days and adjusted } \\
\text { by rate of resistance at admission }\end{array}$ & 0.278 & 0.228 & 0.187 & 0.153 \\
\hline \multirow[t]{4}{*}{ Tobramycin } & At admission & $17(6.0)$ & $32(11.2)$ & $34(11.9)$ & $68(23.9)$ \\
\hline & Acquired in ICU & $1(0.4)$ & $3(1.1)$ & $15(5.3)$ & $11(3.9)$ \\
\hline & Acquired in ICU, by 1000 days & 0.108 & 0.350 & 1.398 & 1.181 \\
\hline & $\begin{array}{l}\text { Acquired in ICU, by } 1000 \text { days and adjusted } \\
\text { by rate of resistance at admission }{ }^{a}\end{array}$ & 0.144 & 0.162 & 0.182 & 0.205 \\
\hline
\end{tabular}

ICU Intensive care unit

The increasing rate of colistin- and tobramycin-acquired colonization resistance in the ICU by 1000 days and adjusted by the rate of resistance at admission was $0.82(95 \% \mathrm{Cl}, 0.56$ to 1.95 ; not statistically significant [NS]). $P$ value for the goodness-of-fit test was 0.427 . For tobramycin, the increasing rate was 1.13 ( $95 \%$ $\mathrm{Cl}, 0.75$ to 1.70 ; nonsignificant). $P$ value for the goodness-of-fit test was 0.159

${ }^{a}$ Adjusted for values corresponding to first year, namely number of patients, number of resistances at admission, and exposure days 


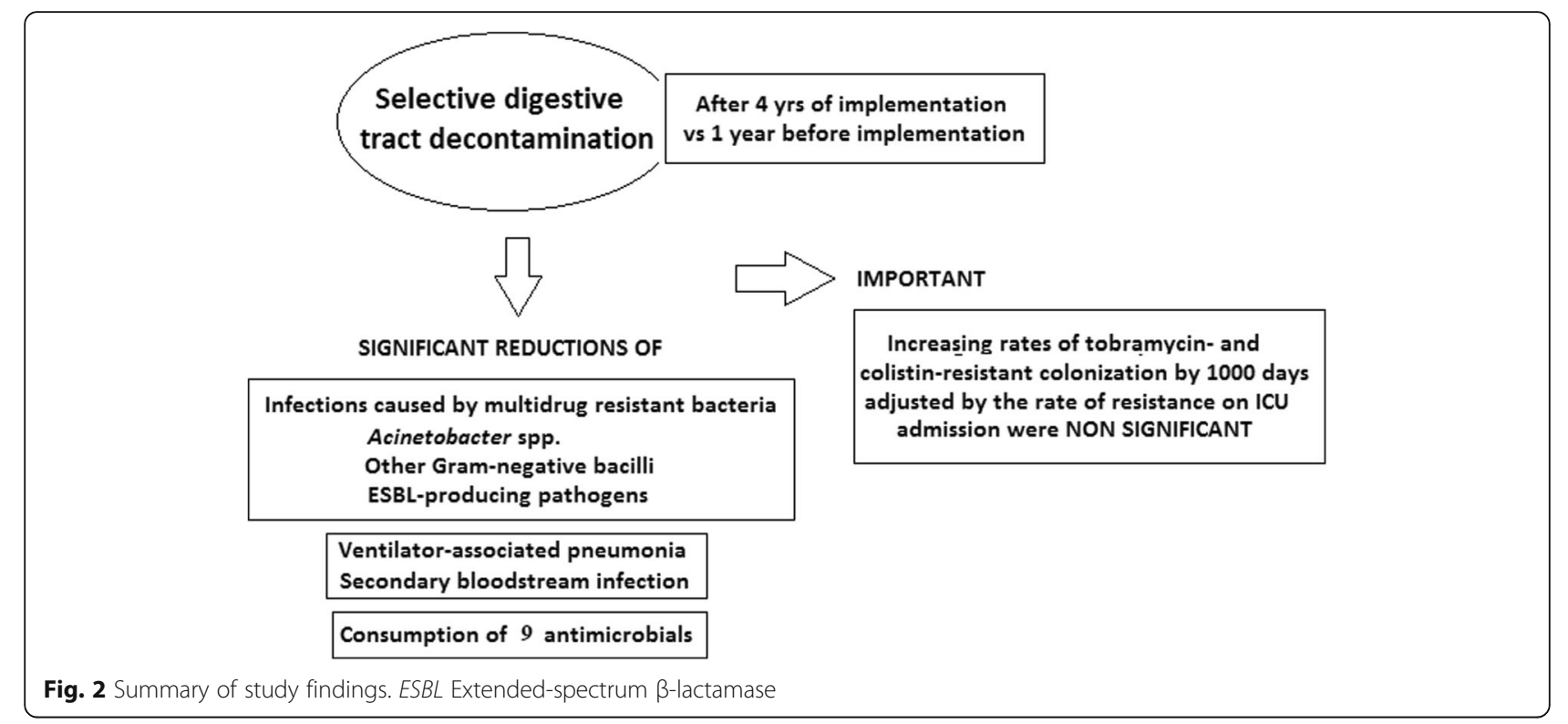

However, the present results are in accord with data of studies carried out in settings with low levels of antibiotic resistance, including findings of systematic reviews of randomized controlled trials [3, 4] and long-term observational studies [24-27], confirming that SDD does not increase resistance. We also observed a significant reduction of infections caused by ESBL-producing MDRB. Similarly, Saidel-Odes and coworkers [28] reported that SDD decreased intestinal overgrowth of carbapenem-resistant $K$. pneumoniae. Zandstra et al. [29] also found that SDD is efficacious in controlling colonization with ESBL-producing bacteria, and Tascini et al. [30] showed that oral administration of gentamicin decontaminated the gastrointestinal tract and prevented infections caused by carbapenem-resistant $K$. pneumoniae strains producing $K$. pneumoniae carbapenemase (KPC)-type $\beta$-lactamase.

We also found a significant reduction of the incidence of infections caused by Acinetobacter baumannii and MDR-GNB. Similarly, in a randomized controlled study of 934 patients admitted to a surgical and medical ICU, of whom 466 were assigned to SDD and 468 to standard treatment (control subjects), colonization with gram-negative bacteria resistant to ceftazidime, ciprofloxacin, imipenem, polymyxin E, or tobramycin occurred in $16 \%$ of SDD patients and in $26 \%$ in the control group $(P=0.001)$ [31]. In a crossover study using cluster randomization in 13 ICUs in the Netherlands, the rate of isolation of gram-negative bacteria from rectal swabs was lower with SDD than with selective oropharyngeal decontamination (SOD) [4]. Also, SDD, as compared with standard care, was associated with a reduction of $57 \%$ of ICU-acquired bacteremia caused by glucose-nonfermenting gram-negative rods $(P$. aeruginosa, Stenotrophomonas maltophilia, and Acinetobacter spp.) and of $81 \%$ by Enterobacteriaceae, and these reductions were not accompanied by increases in intrinsic MDR-GNB colonization or infection [4]. A further analysis showed that development of ICU-acquired bacteremia caused by highly resistant microorganisms was 59\% less frequent with SDD than with standard care and 63\% less frequent with SDD than with SOD [32]. Recently, Camus et al. [33] found that the incidence rate of multidrug-resistant AGNB was lower during SDD (1.59 per 1000 patient-days versus preintervention 5.43\%; $P<0.001$ ) and also declined with time, concluding that a decontamination regimen did not favor the emergence of multidrug-resistant AGNB. In agreement with other studies, infections caused by $C$. difficile [31] and vancomycin-resistant Enterococcus spp. [34] were not registered.

The use of SDD resulted in a significant reduction of VAP, which is consistent with previous observations. In a systematic review of randomized controlled trials of antibiotic prophylaxis in 6914 ICU patients collected from 36 trials, there was a significant reduction of respiratory tract infections in the treated group (OR, 0.28; $95 \% \mathrm{CI}, 0.65$ to 0.87 ) [3]. Also, in a study of 4945 mechanically ventilated patients admitted between 2005 and 2013, the incidence of VAP per 1000 ventilator days declined significantly from $4.38 \pm 1.64$ before to $1.64 \pm 0.43$ after introduction of SOD/ SDD in December $2010(P=0.007)$ [35]. Implementation of SDD as the standard of care in ICUs is thus effective in preventing VAP.

A further remarkable finding of the study was a significant reduction of secondary BSI associated with the use of SDD. In a randomized study involving 16 Dutch ICUs, the proportion of ICU-acquired bacteremia by $\mathrm{En}$ terobacteriaceae was lower for SDD than for SOD (OR, 0.38; $95 \% \mathrm{CI}, 0.26$ to $0.55 ; P<0.001$ ) [1]. In a systematic 
review of 51 randomized controlled trials conducted between 1987 and 2005, comprising 4079 patients treated with SDD and 3986 control subjects, SDD was associated with a reduction of overall and gram-negative BSIs of $27 \%$ and $61 \%$, respectively, without affecting gram-positive BSIs [2]. Furthermore, prophylactic treatment with SDD was a protective factor for infections caused by MRDB. In a systematic review and meta-analysis of 64 studies assessing the effect of SDD and SOD on antimicrobial resistance, no differences were found in the prevalence of colonization or infection with gram-positive antimicrobial-resistant pathogens (MRSA, vancomycin-resistant enterococci) and gram-negative bacilli resistant to aminoglycosides and fluoroquinolones [36]. However, there was a reduction in polymyxin-resistant and third-generation cephalosporin-resistant gram-negative bacilli in recipients of SDD compared with those who did not receive the intervention. According to these data, the perceived risk of long-term harm related to SDD cannot be justified. The authors also conclude that the effect of SDD on ICU-level antimicrobial resistance rates is probably understudied. However, emergence of antimicrobial resistance is still a main objection to the widespread use of SDD in ICUs $[5,6,8]$.

Also, there is a controversy regarding the emergence of an increased resistance to colistin and tobramycin used as part of SDD. We found low rates of colistin- and tobramycin-resistant colonization in cultures of surveillance samples during the 4-year SDD. It is known that there may be nosocomial transmission of highly resistant microorganisms from one patient infected to another, with or without SDD, and that this can increase the number of patients with GNB-resistant colonization [37]. As shown in Table 5, there are increases of colonization resistance to colistin and tobramycin at ICU admission. Also, the estimated rates adjusted to 100 patients with SDD decreased in the fourth year for tobramycin-resistant colonization and showed a small increase from 1.6 to 1.8 for colistin-resistant colonization in the third and fourth years of the study. The colistin- and tobramycin-acquired increasing rates of colonization resistance in the ICU by 1000 days and adjusted by the rate of resistances at admission were 0.82 (95\% CI, 0.56 to 1.95 ; NS) and 1.13 (95\% CI, 0.75 to 1.70; NS), respectively. These findings mean that although there were increases in the rates of colistin- and tobramycin-resistant colonization, these increases could not be associated with SDD and may have been linked to the progressive rise of MDR-GNB at ICU admission over the 4 years of the study and also may have been due to a higher degree of nosocomial transmission of highly resistant microorganisms among ICU patients. The highest estimated rates of colistin- and tobramycin-resistant colonization by 1000 days at risk were 1.2 and 1.1 per 1000 days, respectively (Table 6).
Colistin- and tobramycin-resistant colonization rates in our study were lower than 2.5/1000 patients days at risk, as shown in the study by Oostdijk et al. [38]. Using two large cohorts of ICU patients, Oostdijk et al. demonstrated that the prolonged use of colistin, as part of SDD and SOD, was not associated with increased acquisition of colistin-resistant GNB in the respiratory tract. Moreover, acquisition rates of colistin-resistant GNB in the intestinal tract during SDD ranged from 1.2 to 3.2 per 1000 patient-days at risk. The overall conversion rate from colistin susceptibility to resistance in the intestinal tract was below 1 conversion per 1000 patient-days at risk. During SDD, though, these conversion rates ranged from 3.2 to 5.4 per 1000 days of colonization with GNB and from 15.5 to 12.6 per 1000 days of colonization with tobramycin-resistant GNB. Also, the use of meropenem appeared to be strongly associated with the development of meropenem resistance in $P$. aeruginosa with an adjusted HR of 11.1 (95\% CI, 2.4-51.5), corresponding to 23 events of resistance acquisition per 1000 patient-days at risk. [39]. On the basis of these findings, we concluded, as Oostdijk et al. [38] did, that the rates of resistance acquisition for frequently used antibiotics were considerably higher than for acquisition of colistin resistance during topical use of this agent.

Our findings differ from those of previous studies showing no increase in acquisition of resistant flora to these agents over a 5-year period [24] or no increases in the prevalence of resistance against colistin and tobramycin among gram-negative isolates during a mean of 7 years of SDD or SOD use [40]. Noteboom et al. [41] also observed that the percentages of antibiotic resistance with SDD and standard care were similar.

However, in a short course of SDD with colistin and gentamicin during an outbreak due to a KPC-2-producing $K$. pneumoniae strain, development of secondary resistance to colistin (19\% increase in resistance rate) and gentamicin (45\% increase) was found [8]. Halaby et al. [5] reported a significant relationship between use of SDD and tobramycin resistance as well as resistance to colistin among ESBL-producing pathogens. Brink et al. [6] showed the emergence of KPC in Enterobacteriaceae and the selection of strains resistant to colistin. Of note, Silvestri et al. [42], regarding data reported by Brink et al. [6], argued that an inadequate dose of enteral antimicrobials in the SDD protocol was responsible for the failure of $K$. pneumoniae to decolonize and eventually become resistant to colistin. Failure associated with subtherapeutic doses of SDD may cause overgrowth of MDR-GNB, with increased spontaneous mutation leading to polyclonality and resistance [43].

Associations between prolonged intravenous colistin use and development of colistin resistance have been 
reported from settings with high levels of carbapenemase-producing GNB [44, 45]. In contrast to facilitating resistance, SDD has been used successfully as a control measure in outbreak situations with ESBL-producing GNB [7, 46]. High intraluminal levels of topical antibiotics exceed minimum inhibitory concentrations of resistant pathogens, leading at least to temporary suppression, which reduces the risk of overgrowth and cross-transmission. However, there are several factors aside from SDD that produce GNB-resistant colonization. We did not find any MDR-GNB susceptible only to colistin in our study. Also, we observed decreased ICU global mortality over the course of the 4-year application of SDD.

Nevertheless, we think that SDD must be accompanied by careful monitoring of tobramycin and colistin resistance in GNB. We do so, as described in our protocol. We recommended screening weekly throughout the ICU stay.

\section{Conclusions}

SDD in an ICU setting with a high level of resistance was associated with a clinically relevant reduction of infections caused by MDRB, with low rates of colistin- and tobramycin-resistant colonization and a nonsignificant increasing rate of ICU colonization resistance by 1000 days, adjusted by the rate of resistance at ICU admission. SDD was also a protective factor against MDRB infection. Furthermore, VAP and secondary BSI were significantly decreased after SDD. Notably, a decrease in antimicrobial consumption was also observed.

\section{Abbreviations \\ AGNB: Aerobic gram-negative bacilli; APACHE: Acute Physiology and Chronic Health Evaluation; BSI: Bloodstream infection; CLABSI: Central line-associated bloodstream infection; CLSI: Clinical and Laboratory Standards Institute; CT: Computed tomographic; CVC: Central venous catheter; ENVIN: National Nosocomial Infection Surveillance Study; ESBL: Extended-spectrum $\beta$ - lactamase; EUCAST: European Committee on Antimicrobial Susceptibility Testing; GNB: Gram-negative bacilli; HELICS: Hospitals in Europe Link for Infection Control through Surveillance; ICU: Intensive care unit; KPC: Klebsiella pneumoniae carbapenemase; MDRB: Multidrug-resistant bacteria; MDR- GNB: Multidrug-resistant gram-negative bacilli; MRSA: Methicillin-resistant Staphylococcus aureus; MV: Mechanical ventilation; NS: Nonsignificant; RR: Risk ratio; SDD: Selective digestive tract decontamination; SEMICYUC: Spanish Society of Critical Care Medicine and Coronary Units; SOD: Selective oropharyngeal decontamination; VAP: Ventilator-associated pneumonia; WBC: White blood cells}

\section{Acknowledgements}

The authors thank Marta Pulido, MD, for editing the manuscript and for editorial assistance.

This study was awarded as one of the best communications in the 29th Annual Congress of the European Society of Intensive Care Medicine, Milan, Italy, October 1-5, 2016.

\section{Availability of data and materials}

Please contact the authors for data requests.

\section{Authors' contributions}

CSR designed the study, drafted the manuscript, collected data, analyzed results, and discussed and supervised the registry. SHE collected and analyzed data, critically reviewed the manuscript, and supervised the registry.
MAHV collected data and supervised the registry. LCB collected data and supervised the registry. PS performed statistical analysis and interpreted data. NSM collected data and critically reviewed the manuscript. FAC collected data and critically reviewed the manuscript. CFLV collected data. SRS designed the study, drafted the manuscript, analyzed results, interpreted data, and provided general supervision of the study. MCS collection of data and supervision of the registry. All authors read and approved the final manuscript.

\section{Ethics approval and consent to participate}

The ENVIN registry was approved by the ethics committees of the majority of participating ICUs, including our hospital, and was declared a registry of healthcare interest by the Spanish Ministry of Health, Social Services and Equality in 2014

\section{Consent for publication}

Not applicable, given the noninterventional nature of the study, because data were collected from the ENVIN-HELICS registry.

\section{Competing interests}

The authors declare that they have no competing interests.

\section{Publisher's Note}

Springer Nature remains neutral with regard to jurisdictional claims in published maps and institutional affiliations.

\section{Author details}

${ }^{1}$ Intensive Care Unit, Hospital Universitario de Gran Canaria Dr. Negrín, Las Palmas de Gran Canaria, La Ballena s/n, E-35010 Las Palmas, Spain. ${ }^{2}$ Mathematics Department, Universidad de las Palmas de Gran Canaria, Las Palmas, Spain. ${ }^{3}$ Microbiology Department, Hospital Universitario de Gran Canaria Dr. Negrín, Las Palmas de Gran Canaria, Las Palmas, Spain. ${ }^{4}$ Pharmacy Department, Hospital Universitario de Gran Canaria Dr. Negrín, Las Palmas de Gran Canaria, Las Palmas, Spain.

Received: 26 February 2018 Accepted: 9 May 2018

Published online: 30 May 2018

\section{References}

1. Oostdijk EAN, Kesecioglu J, Schultz MJ, Visser CE, de Jonge E, van Essen $H E R$, et al. Effects of decontamination of the oropharynx and intestinal tract on antibiotic resistance in ICUs: a randomized clinical trial. JAMA. 2014;312: 1429-37.

2. Silvestri L, van Saene HK, Milanese M, Gregori D, Gullo A. Selective decontamination of the digestive tract reduces bacterial bloodstream infection and mortality in critically ill patients. Systematic review of randomized, controlled trials. J Hosp Infect. 2007;65:187-203.

3. Liberati A, D'Amico R, Pifferi S, Torri V, Brazzi L, Parmelli E. Antibiotic prophylaxis to reduce respiratory tract infections and mortality in adults receiving intensive care. Cochrane Database Syst Rev. 2009;4:CD000022.

4. de Smet AM, Kluytmans JA, Cooper BS, Mascini EM, Benus RF, van der Werf TS, et al. Decontamination of the digestive tract and oropharynx in ICU patients. N Engl J Med. 2009;360:20-31.

5. Halaby T, Al Naiemi N, Kluytmans J, van der Palen J, Vandenbroucke-Grauls CM. Emergence of colistin resistance in Enterobacteriaceae after the introduction of selective digestive tract decontamination in an intensive care unit. Antimicrob Agents Chemother. 2013;57:3224-9.

6. Brink AJ, Coetzee J, Corcoran C, Clay CG, Hari-Makkan D, Jacobson RK, et al. Emergence of OXA-48 and OXA-181 carbapenemases among Enterobacteriaceae in South Africa and evidence of in vivo selection of colistin resistance as a consequence of selective decontamination of the gastrointestinal tract. J Clin Microbiol. 2013;51:369-72.

7. Brun-Buisson C, Legrand P, Rauss A, Richard C, Montravers F, Besbes M, et al. Intestinal decontamination for control of nosocomial multiresistant gramnegative bacilli: study of an outbreak in an intensive care unit. Ann Intern Med. 1989;110:873-81.

8. Lübbert C, Faucheux S, Becker-Rux D, Laudi S, Dürrbeck A, Busch T, et al. Rapid emergence of secondary resistance to gentamicin and colistin following selective digestive decontamination in patients with KPC-2-producing Klebsiella pneumoniae: a single-centre experience. Int J Antimicrob Agents. 2013;42:565-70. 
9. Álvarez-Lerma F, Palomar-Martínez M, Sánchez-García M, Martínez-Alonso M, Álvarez-Rodríguez J, Lorente L, et al. Prevention of ventilator-associated pneumonia: the multimodal approach of the Spanish ICU "Pneumonia Zero" program. Crit Care Med. 2018;46:181-8.

10. Wittekamp BH, Ong DS, Cremer OL, Bonten MJ. Nystatin versus amphotericin B to prevent and eradicate Candida colonization during selective digestive tract decontamination in critically ill patients. Intensive Care Med. 2015;41:2235-6.

11. Silvestri L, van Saene HK, Milanese M, Fontana F, Gregori D, Oblach L, Piacente N, Blazic M. Prevention of MRSA pneumonia by oral vancomycin decontamination: a randomized trial. Eur Respir J. 2004;23:921-6.

12. Spanu T, Sanguinetti M, Ciccaglione D, D'Inzeo T, Romano L, Leone F, Fadda G. Use of the VITEK 2 system for rapid identification of clinical isolates of Staphylococci from bloodstream infections. J Clin Microbiol. 2003:41:4259-63.

13. Clinical and Laboratory Standards Institute (CLSI). M100 Performance Standards for Antimicrobial Susceptibility Testing, 27th edition. https:// clsi.org/standards/products/microbiology/documents/m100/. Accessed October 5, 2017.

14. European Committee on Antimicrobial Susceptibility Testing (EUCAST), European Society of Clinical Microbiology and Infectious Diseases. Clinical breakpoints. http://www.eucast.org/clinical_breakpoints/. Accessed October 5, 2017.

15. de La Cal MA, Cerdá E, García-Hierro P, Lorente L, Sánchez-Concheiro M, Díaz C, et al. Pneumonia in patients with severe burns: a classification according to the concept of the carrier state. Chest. 2001;119:1160-5.

16. Sociedad Española de Medicina Intensiva, Crítica y Unidades Coronarias (SEMICYUC), Grupo de Trabajo de Enfermedades Infecciosas. Estudio Nacional de Vigilancia de la Infección Nosocomial (ENVIN): manual de definiciones y términos. http://hws.vhebron.net/envin-helics/Help/Manual_ 2017.pdf. Accessed October 5, 2017.

17. Garner JS, Jarvis WR, Emori TG, Horan TC, Hughes JM. CDC definitions for nosocomial infections, 1988. Am J Infect Control. 1988;16:128-40.

18. Sociedad Española de Medicina Intensiva, Grupo de Trabajo de Enfermedades Infecciosas (SEMICYUC-GTEI). Estudio Nacional de Vigilancia de Infección Nosocomial en UCI (ENVIN-UCl): informes de los años 20012015. http://hws.vhebron.net/envin-helics/index.asp. Accessed March 2018.

19. Dean $\mathrm{CH}$, Lawless JF. Test for detecting overdispersion in Poisson regression models. J Am Stat Assoc. 1989;84:467-72.

20. R Development R Core Team. R: a language and environment for statistical computing. Vienna, Austria: R Foundation for Statistical Computing; 2016. https://www.R-project.org/. Accessed October 5, 2017.

21. Taylor ME, Oppenheim BA. Selective decontamination of the gastrointestinal tract as an infection control measure. J Hosp Infect. 1991;17:271-8.

22. Decré $\mathrm{D}$, Gachot B, Lucet JC, Arlet G, Bergogne-Bérézin E, Régnier B. Clinical and bacteriologic epidemiology of extended-spectrum beta-lactamaseproducing strains of Klebsiella pneumoniae in a medical intensive care unit. Clin Infect Dis. 1998;27:834-44.

23. Agustí C, Pujol M, Argerich MJ, Ayats J, Badia M, Domínquez MA, et al. Short-term effect of the application of selective decontamination of the digestive tract on different body site reservoir ICU patients colonized by multi-resistant Acinetobacter baumannii. J Antimicrob Chemother. 2002;49:205-8.

24. Ochoa-Ardila ME, García Cañas A, Gómez-Mediavilla K, González-Torralba A Alía I, García-Hierro P, et al. Long term use of selective decontamination of the digestive tract does not increase antibiotic resistance: a 5-year prospective cohort study. Intensive Care Med. 2011;37:1458-65.

25. Heininger A, Meyer E, Schwab F, Marschal M, Unertl K, Krueger WA. Effects of long-term routine use of selective digestive decontamination on antimicrobial resistance. Intensive Care Med. 2006:32:1569-76.

26. Leone M, Albanese J, Antonini F, Nguyen-Michel A, Martin C. Long-term (6year) effect of selective digestive decontamination on antimicrobial resistance in intensive care, multiple-trauma patients. Crit Care Med. 2003;31:2090-5.

27. Houben AJ, Oostdijk EA, van der Voort PH, Monen JC, Bonten MJ, van der Bij AK. Selective decontamination of the oropharynx and the digestive tract, and antimicrobial resistance: a 4 year ecological study in 38 intensive care units in the Netherlands. J Antimicrob Chemother. 2004;69:797-804.

28. Saidel-Odes L, Polachek H, Peled N, Riesenberg K, Schlaeffer F, Trabelsi Y, et al. A randomized, double-blind, placebo-controlled trial of selective digestive decontamination using oral gentamicin and oral polymyxin E for eradication of carbapenem-resistant Klebsiella pneumoniae carriage. Infect Control Epidemiol. 2012;33:14-9.
29. Zandstra D, Abecasis F, Taylor N, Damjanovic V, Silvestri L, van Saene HK. For control of colonisation with extended-spectrum $\beta$-lactamase-producing bacteria, SDD does work. Intensive Care Med. 2013;39:539.

30. Tascini C, Sbrana F, Flammini S, Tagliaferri E, Arena F, Leonildi A, et al. Oral gentamicin gut decontamination for prevention of KPC-producing Klebsiella pneumoniae infections: relevance of concomitant systemic antibiotic therapy. Antimicrob Agents Chemother. 2014;58:1972-6.

31. de Jonge E, Schultz MJ, Spanjaard L, Bossuyt PM, Vroom MB, Dankert J, et al. Effects of selective decontamination of digestive tract on mortality and acquisition of resistant bacteria in intensive care: a randomized controlled trial. Lancet. 2003:362:1011-6.

32. de Smet AM, Kluytmans JA, Blok HE, Mascini EM, Benus RF, Bernards AT, et al. Selective digestive decontamination and selective oropharyngeal decontamination and antibiotic resistance in patients in intensive-care units: an open-label, clustered group-randomized, crossover study. Lancet Infect Dis. 2011;11:372-80.

33. Camus C, Sauvadet E, Tavenard A, Piau C, Uhel F, Bouju P, et al. Decline of multidrug-resistant Gram negative infections with the routine use of a multiple decontamination regimen in ICU. J Infect. 2016;73:200-9.

34. Salgado CD, Giannetta ET, Farr BM. Failure to develop vancomycin-resistant Enterococcus with oral vancomycin treatment of Clostridium difficile. Infect Control Hosp Epidemiol. 2004:25:413-7.

35. Schnabel RM, Scholte JB, Van Der Velden KE, Roekaerts PM, Bergmans DC. Ventilator-associated pneumonia rates after introducing selective digestive tract decontamination. Infect Dis (Lond). 2015;47:650-3.

36. Daneman N, Sarwar S, Fowler A, Cuthbertson BH. Effect of selective decontamination on antimicrobial resistance in intensive care units: a systematic review and meta-analysis. Lancet Infect Dis. 2013;13:328-41.

37. Kluytmans-Vandenbergh MF, Kluytmans JA, Voss A. Dutch guideline for preventing nosocomial transmission of highly resistant microorganisms (HRMO). Infection. 2005;33:309-13.

39. Ong DS, Jongerden IP, Buiting AG, Leverstein-van Hall MA, Speelberg B, Kesecioglu J, et al. Antibiotic exposure and resistance development in Pseudomonas aeruginosa and Enterobacter species in intensive care units. Crit Care Med. 2011;39:2458-63.

38. Oostdijk EA, Smits L, de Smet AM, Leverstein-van Hall MA, Kesecioglu J, Bonten MJ. Colistin resistance in gram-negative bacteria during prophylactic topical colistin use in intensive care units. Intensive Care Med. 2013:39:653-60

40. Wittekamp BH, Oostdijk EA, de Smet AM, Bonten MJ. Colistin and tobramycin resistance during long-term use of selective decontamination strategies in the intensive care unit: a post hoc analysis. Crit Care. 2015;19:113.

41. Noteboom Y, Ong DS, Oostdijk EA, Schultz MJ, de Jonge E, Purmer I, et al. Antibiotic-induced within-host resistance development of gram-negative bacteria in patients receiving selective decontamination or standard care. Crit Care Med. 2015;43:2582-8.

42. Silvestri L, Negri C, Taylor N, Zandstra DF, van Saene HK. Inappropriate dose of enteral antimicrobials promotes resistance. J Clin Microbiol. 2013;51:1644.

43. van Saene HK, Taylor N, Damjanovic V, Sarginson RE. Microbial gut overgrowth guarantees increased spontaneous mutation leading to polyclonality and antibiotic resistance in the critically ill. Curr Drug Targets. 2008:9:419-21.

44. Antoniadou A, Kontopidou F, Poulakou G, Koratzanis E, Galani I, Papadomichelakis E, et al. Colistin-resistant isolates of Klebsiella pneumoniae emerging in intensive care unit patients: first report of a multiclonal cluster. I Antimicrob Chemother. 2007:59:786-90.

45. Matthaiou DK, Michalopoulos A, Rafailidis PI, Karageorgopoulos DE, Papaioannou V, Ntani G, et al. Risk factors associated with the isolation of colistin-resistant gram-negative bacteria: a matched case-control study. Crit Care Med. 2008:36:807-11

46. Buehlmann M, Bruderer T, Frei R, Widmer AF. Effectiveness of a new decolonization regimen for eradication of extended-spectrum $\beta$-lactamaseproducing Enterobacteriaceae. J Hosp Infect. 2011;77:113-7. 